\title{
Synergy between two transcription factors directs gene expression in Dictyostelium tip-organiser cells
}

\author{
HONG YU WANG and JEFFREY G. WILLIAMS* \\ College of Life Sciences, University of Dundee, U.K.
}

\begin{abstract}
C$ requires the transcription factor CudA for its expression in the posterior, prespore cells of the slug, while the expL7 gene requires CudA for its expression in the anterior, tip-organiser region. In order to identify additional transcription factors that might mediate tiporganiser specific expression, we performed affinity chromatography on slug nuclear extracts. The affinity matrix bore cap-site distal sequences from region $A^{\prime}$ of the expL7 promoter; an essential region located upstream of the CudA binding domain. One of the proteins purified was G-box binding factor (GBF), a zinc finger transcription factor which binds to G-rich elements, known as $G$ boxes, that are present in the promoters of many developmental genes, including cotC. Previous work identified an essential sequence motif within region $A^{\prime}$ and we show that this element is a $\mathrm{G}$ box, that binds recombinant GBF. Moreover, a $\mathrm{G}$ box from within the cotC promoter can substitute for region $A^{\prime}$ of $\exp L 7$ in directing tip-organiser specific expression of exp $L 7$. Thus the same two transcription factors, CudA and GBF, seem to co-operate to direct both tip-organiser and prespore gene expression. How then is specificity achieved? Replacing a CudA binding region in the $\cot C$ promoter with the CudA binding domain from expL7 strongly represses cotC promoter activity. Hence we suggest that differences in the topology of the multiple CudA half- sites contained within the two different CudA binding regions, coupled with differences in the signalling environment between tip-organiser cells and prespore cells, ensure correct $\operatorname{expL7}$ expression.
\end{abstract}

KEY WORDS: Dictyostelium, cudA, GBF, tip-organiser, prespore, transcription factor

Dictyostelium develops to form a fruiting body comprised of just two terminally differentiated cell types, stalk and spore cells, but, at the preceding slug stage, prespore cells and several sub-types of prestalk cell can be recognised. The cells of one of the prestalk sub-types constitute the tip-organiser, a tissue that behaves rather like an embryonic organiser and that also directs slug behaviour (Raper, 1940; Poff and Loomis, 1973; Rubin and Robertson, 1975; Smith and Williams, 1980).

CudA is the founder member of a class of transcription factors that are present only in the amoebozoa (Fukuzawa et al., 1997). CudA is expressed and nuclear localised in the tip-organiser cells where it is necessary for the transcription of a direct target gene, $\exp L 7$, that encodes an expansin-like protein (Ogasawara et al., 2009; Wang and Williams, 2009). CudA acts as a secondary transcription factor in a transcriptional cascade; wherein tipspecific accumulation of the ACA adenylyl cyclase mRNA causes localised cAMP synthesis which triggers STATa activation within the tip (Verkerke-van Wijk et al., 2001). STATa then binds to the cudA promoter and directs $c u d A$ transcription. CudA is also expressed and nuclear localised in prespore cells, where it acts as a secondary transcription factor necessary for optimal expression of the $\cot C$ spore coat protein gene (Yamada, et al., 2008).

The expL 7 and $\cot C$ promoters contain binding sites for CudA, that are essential for optimal expression but that are not sufficient to direct expression when linked to minimal promoter elements (Yamada, et al., 2008; Wang and Williams, 2009). Transcription factors generally function co-operatively, with other transcription factors, by binding to synergising promoter elements to achieve the requisite level of gene expression. Often this involves the cooperation of cell-type specific with non cell-type specific transcription factors, such as SP1 (Kadonaga et al., 1987). In the case of

Abbreviations used in this paper: GBF, G-box binding factor.

\footnotetext{
*Address correspondence to: Jeffrey G. Williams. College of Life Sciences. University of Dundee, Dow St., Dundee DD1 5EH, UK. e-mail: j.g.williams@dundee.ac.uk
} 
post-aggregative Dictyostelium development, a similar role is frequently played by GBF (G-box binding factor).

GBF contains two putative zinc fingers and binds to GT- or, in the opposite strand, CA-rich elements (both relative orientations will be termed $\mathrm{G}$ boxes, unless referring to a previously named element) that are frequently located upstream of post-aggregation genes (Hjorth et al., 1988; Hjorth et al., 1990; Pears and Williams, 1987; Schnitzler et al., 1994). G-boxes are essential for optimal gene transcription within the context of their own promoter but are inactive when linked to basal promoter elements. The cotC promoter contains three $\mathrm{G}$ boxes, termed $\mathrm{CA}$-rich elements or CAEs (Powell-Coffman et al., 1994). They synergise with a downstream TA-rich region to help direct efficient $\operatorname{cotC}$ expression (Powell-Coffman et al., 1994). Additionally, prespore specificity is endowed by a CudA binding region, termed region $B$, located between the two cap-site proximal CAEs (Yamada, et al., 2008).

For unknown reasons, recombinant forms of CudA fail to bind at specific promoter sites, hence an Entamoeba orthologue with similar apparent specificity, ECudA, is used for mapping promoters (Yamada, et al., 2008). CudA exists as a dimer and region $B$ of cotC contains a complete ECudA binding site, the dyad GAATTTTC, and a more proximal half site, GAA. The expL7 promoter (Fig. 1A) contains an approximately 100nt ECudA binding region, also called region $B$, that is essential for tipspecific expression (Wang and Williams, 2009). This region contains several dispersed sub-regions that are necessary for maximal binding by ECudA. There are four potential TTC CudA half-sites within region $B$ but, because of their partial mutual redundancy, they were not precisely delineated by mutation (Wang and Williams, 2009).
CudA is, as stated, present in the nuclei of both the tiporganiser and the prespore cells and is essential for exp $\angle$ and $\cot C$ expression. What then prevents exp $\angle$ from being expressed in the prespore cells and cotCbeing expressed in the tiporganiser cells; is there an additional transcription factor that confers discrimination between the two promoters? Region $A$ (Fig. $1 \mathrm{~A}$ and $2 \mathrm{~A}$ ) of the exp $\angle 7$ promoter is a candidate for the binding site of such a factor. Region A does not bind CudA but is required for expL 7expression in tip organiser cells (Ogasawara et al., 2009; Wang and Williams, 2009). An essential CA-rich sequence element has been identified in region $A$ by mutational analysis and was proposed to be a site of binding for the DIFregulated bZIP protein, DimB (Ogasawara etal., 2009). However, no direct evidence was presented for this. In order to determine whether region A might contain ancillary regulatory elements that confer specificity on $\exp L 7$, we undertook an analysis of the proteins that bind to it.

\section{Results}

\section{Two known transcription factors are purified by cudA pro- moter region $A^{\prime}$}

Region A of the expL 7promoter contains two relatively GC-rich tracts, separated by a $65 \mathrm{nt}$ region comprised entirely of $A$ and $T$ residues (Fig. $1 \mathrm{~A}$ and $\mathrm{Fig}$. 2A). The essential sequences of region A lie in the cap-site distal GC-rich region, region A' (Ogasawara et al., 2009). Hence slug nuclear extracts were purified on an affinity resin bearing the 43nt sequence comprising region A' (Fig. 1A). Multiple proteins were bound (Fig. 1B) but most are either RNA binding proteins, a class of proteins that is often purified in such experiments (Fukuzawa, et al., 2006), or previously un-

A
expL7region $A$
$-823$ expL7region $A$
AAATAGTTTTTAATGTGGTATTTCACACAGTCTATAGAC(65A expL7region A'mut AAATAGTTTTTAATGTGGTATTTGCGCGCGTCTATAGACTTTT cotC CAE-2
CAE- consensus TATTCATTCACACATTAACACACTTTCAACTCAATTTT CACACAYYY-CACACAAA/T

B

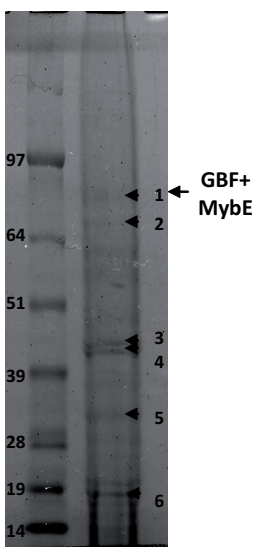

C Dictyostelium nuclear extract
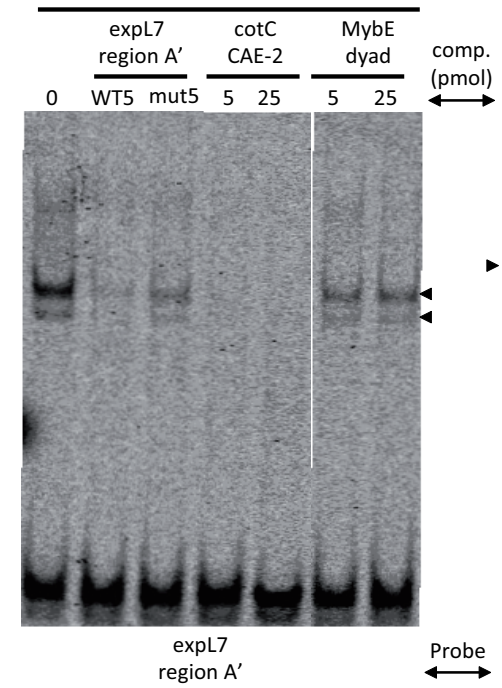

D

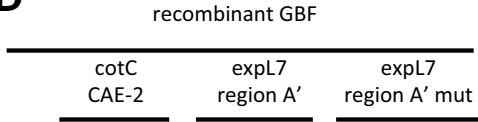

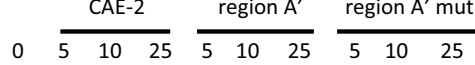

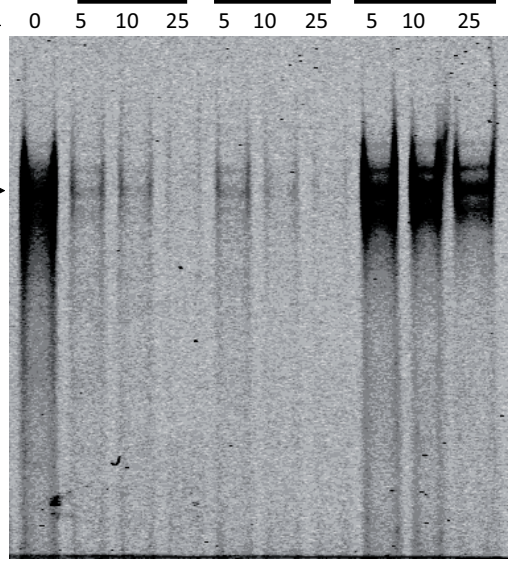

Fig. 1. Affinity purification of nuclear proteins that bind to region $A^{\prime}$ and band-shift analysis of proteins interacting with region $\mathbf{A}^{\prime}$. (A) An alignment, in red, of the CACACA sequence in CAE-2 with the CACACA sequences in region $A^{\prime}$ of expL7. Note that the cap-site proximal GC-containing tract contains a CACACA sequence in its invert complement. Only the distal part of the proximal sequence is shown. It extends another 30nt, with the sequence TGAATTATTATTTATTTGTTTTTATTTATT. (B) Total nuclear extracts were subjected to affinity chromatography on $A^{\prime}$ and analysed by mass spectrommetry as described in Methods. Slice 1 contained the only two recognisable transcription factors: MybE (93kDa) and GBF (79kDa). We assume that we are detecting a large degradation product of MybE because slice 1 is tightly centred around a nominal mol wt of $80 \mathrm{kD}$. (C) Region A' was labeled and used as probe in gel retardation with heparin purified slug nuclear extract and with the indicated competitors. (D) CAE-2 was labeled and used as probe in gel retardation with GBF produced in $E$. coli and with the indicated competitors. 
known proteins. There were just two recognisable transcription factors, GBF and MybE. Both were contained within slice 1, the gel slice bearing the largest proteins analysed (Fig. 1B). GBF contains two potential zinc fingers and has a predicted molecular weight of $79 \mathrm{kDa}$ (Schnitzler, 1993). MybE is a single Myb domain SHAQKY family protein (Fukuzawa etal., 2006). It has a predicted molecular weight of $93 \mathrm{kDa}$ and is required for DIF-inducible gene expression and correct prestalk cell differentiation.

\section{There is a binding site for G-box binding factor (GBF) within region $A^{\prime}$}

When region $A^{\prime}$ is used as a probe in a band shift assay with heparin purified nuclear extracts, there is a major retarded band and a minor, faster-migrating band (Fig. 1C). The two retarded complexes apparently contain GBF; because CAE-2, a wellcharacterised $\mathrm{G}$ box located within the $\cot C$ promoter, is a more potent competitor for binding to the A' probe than is A' itself (Fig. $1 \mathrm{C})$. MybE was also purified in the affinity chromatography but an oligonucleotide sequence from the ecmA promoter containing the MybE dyad does not compete for binding to region A' (Fig. 1C). Also there is no obvious fit to the consensus MybE dyad binding site, AACnGTT, within region $A^{\prime}$. MybE could be present as a contaminant in the eluate from the affinity resin or it may bind via an interaction with another protein present in the complex. It was not investigated further.

The consensus site for the binding of GBF to a CAE is CACACAYYYCACACAAA/ $\mathrm{T}$ (Powell-Coffman et al., 1994) and this region of $C A E-2$ is indicated in red in Fig. $1 A$. In region $A^{\prime}$ there is a sequence with perfect homology to the distal half of the CAE sequence, CACACA, but there is only limited homology to the proximal CACACA element. However, downstream of the long AT tract there is a CACACA sequence in the complementary strand (red in Fig. 1A). GBF functions by binding co-operatively to multiple $\mathrm{G}$ box elements, independent of their relative orientation (Pears and Williams, 1988). Hence the downstream sequence could subsume the function of the proximal CACACA element. We mutated the entire distal CACACA element, as indicated in Fig. $1 A$, and assayed relative competition activity in a band-shift assay using region $A^{\prime}$ as the probe (Fig. 1C). The mutant form, $A^{\prime}$ mut, is a significantly poorer competitor than region $A^{\prime}$ itself (Fig. $1 \mathrm{C}$ ).

The above band shift assay supports the notion that GBF binds to the expL 7 promoter and maps the binding activity to the CACACA sequence. There are, however, close homologues of GBF in Dictyostelium and one of these could, in principle, be responsible for the observed binding. We therefore expressed GBF in E. coli as a HIS fusion protein, purified the protein on metal affinity resin, and performed band shift assays using CAE-2 as probe (Fig. 1D). When used as a competitor, region $A^{\prime}$ is as effective as CAE-2 itself. Moreover A' mut, the sequence containing mutations that ablate the CACACA element, is much less effective as a competitor (Fig. 1D). Thus recombinant GBF binds to region A' of the exp $\angle 7$ promoter and it does so by binding to the CACACA element.

The $G$ box is the active element within region A' and a G box derived from cotC can subsume the function of $A^{\prime}$

The full length expl7 promoter construct, -1117 , is expressed throughout the tip-organiser while the fore-shortened, -823 construct is expressed only in the rear part of the tip-organiser (Wang and Williams, 2009 and Fig. 2). The CACA element located in region $A^{\prime}$ has previously been point-mutated in two of its $C$ residues but, because its parent construct is not expressed at the slug stag, an effect of the mutation was only measurable during culmination (Ogasawara et al., 2009). We therefore generated construct $-823 \mathrm{M}$. It is equivalent to construct -823 , which we find to be expressed in the posterior of the tip-organiser region at the

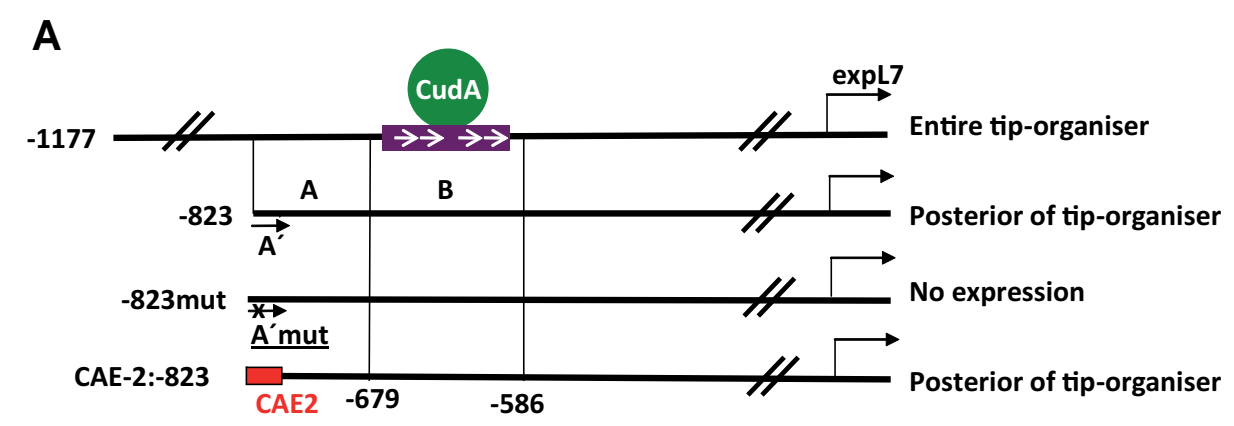

B

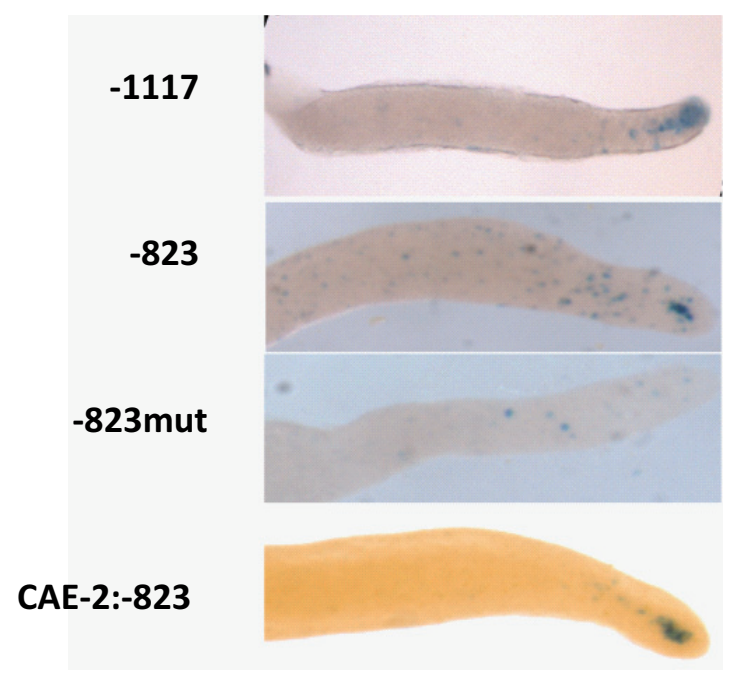

Fig. 2. expL7 promoters reporter fusions and their expression patterns. (A) A representation of the explA7 promoter showing the positions of the four proposed CudA half sites, relative to the previously mapped regions $A, B$ and $C$. Each TTC half site is represented by an arrow. There are also CudA binding sites within region $C$ but they have not been at all mapped to the sequence. In construct -823 mut, region $A^{\prime}$ was mutated to region $A^{\prime}$ mut, the sequence shown in Fig. $1 A$. In construct $C A E-2: 823$, region $A^{\prime}$ was replaced by the $C A E-2$ sequence from the cotC promoter, again the sequence was as in Fig. 1A. (B) Cells transformed with the indicated lacZ reporter constructs (Fig. 2A) were allowed to develop to the slug stage, fixed and stained for $\beta$ galactosidase. 
slug stage (Wang and Williams, 2009) but contains the same 6 nucleotide substitution (CACACA to GCGCGC) that eliminates binding of region $A^{\prime}$ to GBF (Figs. $1 A$ and $2 A$ ). While -823 is expressed in the expected pattern, $-823 \mathrm{M}$ is entirely inactive (Fig. 2B). Thus the CACACA element is essential for tip-organiser expression at the slug stage.

If GBF mediates the essential role that region A' plays in tiporganiser gene expression, it should be possible to replace region $A^{\prime}$ with a generic $\mathrm{G}$ box. We tested this using CAE-2 from $\cot C$. In construct CAE-2:823 expression is restored in the rear half of the tip-organiser region (Fig. 2B). This mirrors the expression pattern observed for construct -823 , where region $A^{\prime}$ is present (Fig. 2B). Collectively these data imply that GBF synergises with CudA to activate expL7 but we cannot, of course, completely rule out the possibility that another transcription factor with a similar specificity binds region $\mathrm{A}^{\prime}$ in vivo.

\section{When transplanted into the cotC promoter, the CudA binding} domain from expL7 acts as a transcriptional inhibitor

The above result shows that region $A$ is required purely because it contains a G-box. Therefore sequences in region $A$ do not dictate that $\exp L 7$ should be expressed in the tip-organiser cells but not the prespore cells. That information must be encoded downstream of region $A$. Region $B$ is essential for tip organiser expression but the only known factor that binds there is CudA and CudA is also necessary for $\cot C$ expression in prespore cells. There could, however, be a binding site for another transcription factor in region $B$. Alternatively, differences in the positions and relative orientation of the multiple CudA binding sites between $\exp L 7$ and $\cot C$ might, in some way, determine their different properties.

To help distinguish the above possibilities, we replaced the known CudA binding region of $\cot C$, region $\mathrm{B}$ in our previous analysis (Yamada, et al., 2008), with region $B$ from expL 7 . The start point was a new construct, $\cot C \Delta \mathrm{B}$; an internal deletion mutant of the full length $\cot C$ promoter, construct CotC, that lacks region $B$ (Fig. 3A). Expression of this and related constructs, all driving expression of a lacZ reporter, was compared in three separate transformant pools: both by $\beta$-galactosidase staining of whole mount slugs and enzymatic assay (Figs. 3 B,C). The latter analysis allows quantitative comparison between the expression levels of the constructs and the use of multiple pools averages out

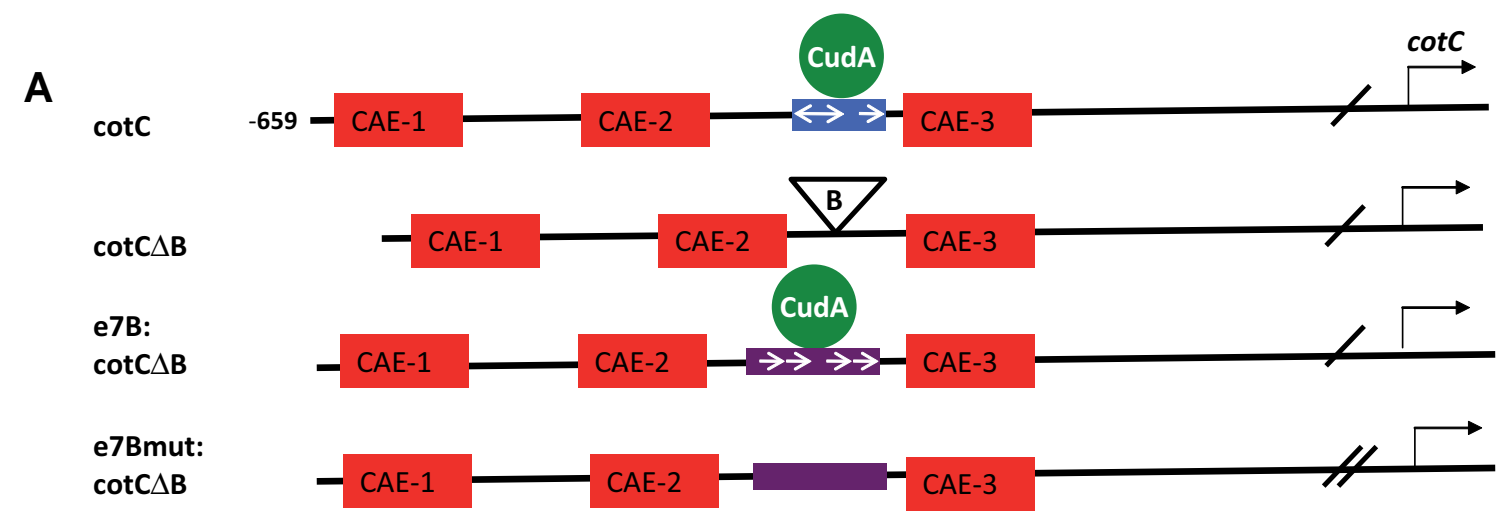

B

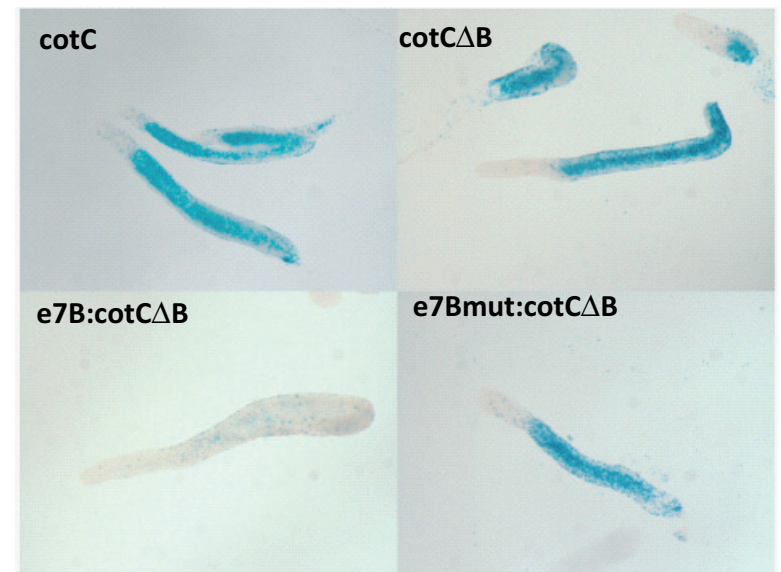

C

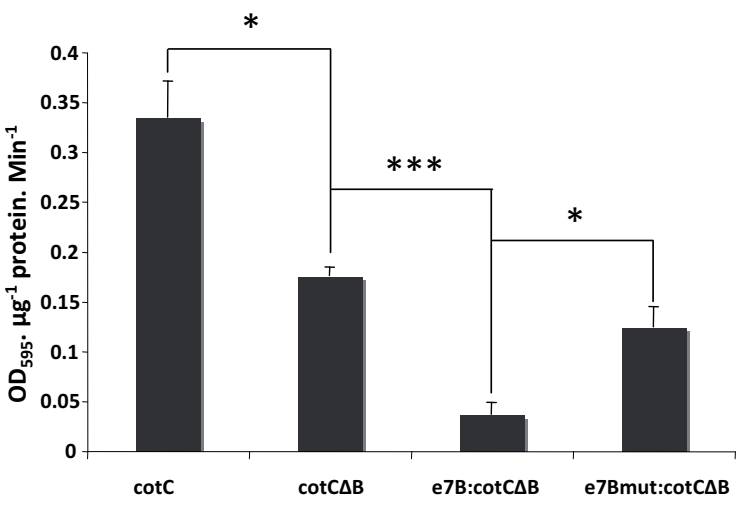

Fig. 3. Insertion of CudA binding region B from expL7into the $\cot C$ promoter. (A) A schematic representation of the cotC promoter and constructs derived from it. The promoter contains three CAE elements, one dyad CudA binding site and one half CudA binding site. Each half site is represented by an arrow. In construct $\cot C \Delta B$ the 66 nucleotide of the $C u d A$ binding region is deleted. Constructs e7B: $\cot C \Delta B$ and e7Bmut:cotC $\Delta B$ were generated from $\cot C \Delta B$ by inserting either wild type or mutant forms of the CudA binding region from the expL7 promoter. (B) Cells transformed with the indicated lacZ reporter constructs (Fig. $3 \mathrm{~A}$ ) were allowed to develop to the slug stage, fixed and stained for $\beta$-galactosidase for $30^{\prime}$ at $37^{\circ} \mathrm{C}$. (C) Cells transformed with the indicated lacZ reporter constructs (Fig. 3A) were developed to the slug stage, protein was isolated and $\beta$-galactosidase enzymatic activity was measured. The average results from three separate transformant pools, assayed in duplicate are shown with S.D. The statistical significance of selected of the results was further assessed using the Student $t$ test and are indicated using asterisks: * $p=0.05$ and $* * 0.001$. 
any effects of copy number.

Despite the deletion, $\cot C \Delta \mathrm{B}$ is still expressed in prespore cells, but at a two-fold lower level than cotC (Fig. 3C, significant at $p=0.05)$. The residual level of expression presumably indicates that there are additional CudA binding sites, located elsewhere in the promoter, that are redundant with those in region $\mathrm{B}$. Most likely, these are in the region -659 to 483; because deletion of that region produces a major drop in prespore gene expression and, in a construct with a distal end-point at -457 , mutation of the three CudA half-sites in region B greatly attenuates expression (Yamada, etal., 2008).

When region $\mathrm{B}$ from $\exp L 7 \mathrm{is}$ inserted into $\cot \mathrm{C} \Delta \mathrm{B}$, to generate $\mathrm{e} 7 \mathrm{~B}: \cot \mathrm{C} \Delta \mathrm{B}$, there is almost no expression in the tip-organiser (Figs. $3 \mathrm{~B}, \mathrm{C}$, significant at $\mathrm{p}=0.001$ ). Hence region $\mathrm{B}$ from $\exp \mathrm{C} 7$ does not carry the sequence information necessary to direct tiporganiser expression, at least when within the context of an otherwise prespore-specific promoter. Interestingly however, it exerts a strong inhibitory effect on prespore expression directed by the residual $\cot C$ promoter elements within $\cot C \Delta \mathrm{B}$ (Figs. 3 $\mathrm{B}, \mathrm{C})$. Construct e7Bmut:cotC $\Delta \mathrm{B}$ contains block mutations; within the three sub-regions known to be essential for optimal CudA binding and that, between them, ablate the four proposed ECudA half-sites (Wang and Williams, 2009). It does not display the same inhibited expression level as e7B:cotC $\Delta \mathrm{B}$ (Figs. $3 \mathrm{~B}, \mathrm{C})$. We are, unfortunately, precluded from performing the symmetrical experiment, to determine whether region $B$ from the cot $C$ gene downregulates expL7 when it is used to replace region $B$ of expl7. In the case of the expL7 promoter, deletion of region $B$ totally prevents tip-specific expression (Wang and Williams, 2009). Therefore, there is no residual expression to be inhibited by adding in regionB from cotC.

\section{Discussion}

The tip-organiser cells are a small sub-set of the prestalk cells that control slug integrity and behaviour. The prespore cells are destined to become spores and differ from the tip-organiser cells in many important ways. Nonetheless, there is a striking similarity in the way two of the markers for these tissues, $\cot C$ and $\exp L 7$, are regulated; both lie at the end of transcriptional cascades involving CudA and both seem to involve a synergy of CudA with GBF. This congruity is most clearly demonstrated by the fact that a $G$ box from $\cot C$ can functionally substitute for the $G$ box of expL 7 .

Such interchangeability of promoter elements has been demonstrated previously; using a G box from the DIF regulated ecmB promoter to replace a $\mathrm{G}$ box located in a cAMP regulated promoter (Ceccarelli et al., 1992). The present observations, again using two genes with radically different expression patterns, re-inforce the notion of GBF as a general transcription factor which cooperates with regulatory transcription factors to facilitate the expression of most, if not all, classes of post-aggregation genes. A "synthetic" reporter construct, containing multimerised CudA dyad binding sites, fused downstream of CAE-2 to basal promoter elements from the Actin 15 gene, was not expressed in a cell type specific manner (Wang and Williams, unpublished data). Hence we cannot rule out the involvement of other transcription factors in either pathway and, indeed, a cap-site proximal AT-rich region, is known to be important for cotCexpression (Powell-Coffman et al., 1994).

A negative result from a promoter shuffling experiment is, of course, intrinsically difficult to interpret; the spacing and configuration of the elements may be incorrect or they may require specific basal promoter sequences. We did obtain interesting information when we attempted the less ambitious experiment of using the CudA binding domain of $\exp L 7$, domain $\mathrm{B}$, to replace the characterised CudA binding domain of $\cot C$. Rather than the expected stimulation of expression, back up to the level of the undeleted construct, there was a strong inhibition. This observation could explain why exp $\angle$ is expressed in the tip-organiser cells but not in the prespore cells.

We propose that within the signalling environment of tiporganiser cells CudA bound to region $B$ of exp 7 functions, in conjunction with GBF, as an activator of $\exp L 7$ transcription (Fig. $4 A)$. Within the signalling environment of prespore cells, either: CudA bound to region $B$ of $\exp L 7$ acts as a repressor of exp 7 transcription (Fig. 4B) or CudA is displaced from the expL 7 promoter and replaced by a transcriptional repressor, designated $X$ in Fig. 4C. The nuclear hormone receptor super-family provides precedents where the configuration of half sites determines regulatory polarity (Naar et al., 1991; Carr and Wong, 1994; Kurokawa et al., 1994; Retnakaran et al., 1994; Jacobsen et al., 2009). Similar differences in the topology of CudA half sites could explain why $\cot C$ shows the opposite beaviour to $\exp L 7$ in prespore cells. The inhibitory effect of exp 7 domainB is dependent upon the presence of the four proposed CudA half sites but this observation has to be interpreted cautiously, because the 
sites have not been precisely mapped. Transcription factors other than CudA (X in Fig. 4C) could share part or all of the CudA binding specificity and could replace CudA to act as the repressor. There are five CudA orthologues in Dictyosteliumand one of these may fulfil that function. The fact that deletion of region $B$ from expL7 does not lead to ectopic expression within the prespore region (Wang and Williams, 2009) perhaps indicates that it also contains sequences important for the activation of expression in prespore cells.

\section{Materials and Methods}

\section{Cell culture and development}

All experiments were performed with the Gerisch isolate of Ax2. Cells were grown, developed, transformed and assayed for lacZ expression as described previously (Fukuzawa and Williams, 2000).

\section{Affinity purification of proteins}

Slug nuclear extracts derived from $4 \times 10^{11}$ slug cells were precipitated with $50 \%$ ammonium sulfate and subjected to heparin-agarose chromotagraphy and DNA affinity chromatography as described previously (Fukuzawa, et al., 2006). Protein was analysed on 4\%-12\% Bis-Tris SDS-polyacrylamide gels and selected regions of the gel were analysed by mass spectrommetry (Fukuzawa, et al., 2006).

\section{Band shift analysis}

The entire GBF coding region was cloned as a "6XHIS" fusion construct in $\mathrm{pET} 15 \mathrm{~b}$ (Novagen, Ltd), expressed in E, coli and purified over TALON $^{T M}$ metal affinity resin (BD Biosciences, Ltd). Band shift analysis was performed as described previously (Wang and Williams, 2009) using oligonucleotides labelled with Cy5-dCTP (Amersham, Ltd).

\section{Generation of promoter constructs}

The -823 exp $\angle$ promoter construct was described previously (Wang and Williams, 2009). The constructs -823M and CAE-2:-823 were generated by cloning double stranded oligonucleotides respectively containing the sequences $A^{\prime}$ mut or CAE-2 (sequences as in Fig. 1A) at their distal ends, coupled to the central 65nt AT sequence and the proximal GCcontaining sequence (Fig. 1A). They were cloned, with GATC cohesive ends into the BamHI site of $\exp L 7$ construct -723; a deletion construct that lacks region $A$ (Wang and Williams, 2009). The $\cot C-659$ construct was described previously (Yamada, et al., 2008). CotC $\Delta \mathrm{B}$ was generated by inserting $\cot C$ promoter fragment -659 to -483 into construct $\cot C-416$ (Yamada, et al., 2008) using $\mathrm{Xbal}$ and $\mathrm{BamHI}$. This resulted in a $66 \mathrm{nt}$ deletion within the promoter. Using $\cot C \Delta \mathrm{B}$ as recipient, $\exp L 7$ region $\mathrm{B}$ in its unmutated or multiply mutated form (sequences shown below) were inserted into the BamH site with GATC cohesive ends to respectively generate e7B: $\cot C \Delta B$ and $e 7 B m u t: \cot C \Delta B$, unmutated domain $B$ : ATTTGTTTTAATTTCTTTATTTTCAATTACCCAAAAGATTATTATACATC ATAATTATTCTATATGATTACGTACACGTTTTTTAAACAATTTCTAA and mutated domain B:ATTTGTTTTAGCGCGCGCGCGCGCGCTTACC CAAAAGATTATTATACATCATAATTAGCGCGCGCGATTACGTACACGTTITTAAACA GCGCGCGC. The positions of the mutations were based on mutation scanning results (Wang and Williams, 2009), where sequential 8 nucleotide blocks of sequence within domain $B$ were replaced with 8 nucleotide alternating $\mathrm{GC}$ tracts. Here the $\mathrm{GC}$ tracts are underlined. The suggested CudA half-sites in the unmutated sequence are in bold.

\section{$\beta$-galactosidase staining and enzymatic activity analysis}

Cells bearing lac $Z$ contructs were developed to the slug stage and either fixed and stained for $\beta$-galactosidase (Dingermann et al., 1989) or harvested in lysis buffer ( $100 \mathrm{mM}$ Hepes, $\mathrm{pH} 8,1 \mathrm{mM} \mathrm{MgSO}_{4}, 5 \mathrm{mM}$ DTT, $2 \%$ Triton X-100). The protein concentration was determined and $5 \mathrm{ul}$ of protein was added to $100 \mathrm{ul}$ of lysis buffer containing $1 \mathrm{mM}$ CPRG. OD was measured at $595 \mathrm{~nm}$ at several time points. LacZ activity is displayed as $\mathrm{OD}_{595}$ per ug protein per minute.

\section{Acknowledgements}

We would like to thank the Wellcome Trust (Project Grant 078971/Z) 05/Z) for funding this work.

\section{References}

CARR F E AND WONG N C., (1994). Characteristics of a negative thyroid hormone response element. J Biol Chem 269: 4175-4179.

CECCARELLI A, MAHBUBANI H. J, INSALL R, SCHNITZLER G, FIRTEL R A AND WILLIAMS J G, (1992). A G-rich sequence element common to Dictyostelium genes which differ radically in their patterns of expression. Dev Bio/152: 188193.

DINGERMANN T, REINDL N., WERNER H, HILDEBRANDT M, NELLEN W, HARWOOD A, WILLIAMS J AND NERKE K (1989). Optimization and in situ detection of Escherichia coli beta-galactosidase gene expression in Dictyostelium discoideum. Gene 85: 353-362.

FUKUZAWA M, HOPPER N AND WILLIAMS J (1997). cudA: A Dictyostelium gene with pleiotropic effects on cellular differentiation and slug behaviour. Develop ment 124: 2719-2728.

FUKUZAWA M, ZHUKOVSKAYA N V, YAMADA, Y, ARAKI T AND WILLIAMS, J G (2006). Regulation of Dictyostelium prestalk-specific gene expression by a SHAQKY family MYB transcription factor. Development 133: 1715-1724

FUKUZAWA M, ZHUKOVSKAYA N V, YAMADA Y, ARAKI T AND WILLIAMS J G (2006). Regulation of Dictyostelium prestalk-specific gene expression by a SHAQKY family MYB transcription factor. Development 133: 1715-1724.

HJORTH A, DATTA S, KHANNA N C AND FIRTEL R A (1988). Analysis of cis and trans elements involved in CAMP-inducible gene expression in Dictyostelium discoideum. Dev Genet 9: 435-454.

HJORTH A L, PEARS C, WILLIAMS J G AND FIRTEL R A (1990). A developmentally regulated trans-acting factor recognizes dissimilar $\mathrm{G} / \mathrm{C}$-rich elements controlling a class of cAMP-inducible Dictyostelium genes. Genes Deve/4: 419432.

JACOBSEN B M, JAMBAL P, SCHITTONE S A AND HORWITZ K B (2009). ALU repeats in promoters are position-dependent co-response elements (coRE) that enhance or repress transcription by dimeric and monomeric progesterone receptors. Mol Endocrino/23: 989-1000.

KADONAGA J T, CARNER K R, MASIARZ F R AND TJIAN R (1987). Isolation of cDNA encoding transcription factor $\mathrm{Sp} 1$ and functional analysis of the DNA binding domain. Cel/51: 1079-1090.

KUROKAWA R, DIRENZO J, BOEHM M, SUGARMAN J, GLOSS B. ROSENFELD M G, HEYMAN R A, AND GLASS C K. (1994). Regulation of retinoid signalling by receptor polarity and allosteric control of ligand binding. Nature $371: 528$ 531.

OGASAWARA S, SHIMADA N AND KAWATA T (2009). Role of an expansin-like molecule in Dictyostelium morphogenesis and regulation of its gene expression by the signal transducer and activator of transcription protein Dd-STATa. Dev Growth Differ 51: 109-122.

PEARS C. J. AND WILLIAMS J. G., (1987. Identification of a DNA sequence element required for efficient expression of a developmentally regulated and cAMP-inducible gene of Dictyostelium discoideum. EMBO J6: 195-200.

PEARS C J AND WILLIAMS J G (1988). Multiple copies of a G-rich element upstream of a cAMP-inducible Dictyostelium gene are necessary but not sufficient for efficient gene expression. Nucl Acids Res 16: 8467-8486.

POFF KL AND LOOMIS WF (1973). Control of phototactic migration in Dictyostelium discoideum. Exp. Cell Res. 82: 236-240.

POWELL-COFFMAN J A, SCHNITZLER G R AND FIRTEL R A (1994). A GBFbinding site and a novel AT element define the minimal sequences sufficient to direct prespore-specific expression in Dictyostelium discoideum. Mol Cell Biol 14: 5840-5849.

RAPER KB (1940). Pseudoplasmodium formation and organization in Dictyostelium discoideum. J. Elisha Mitchell Sci Soc 56: 241-282.

RETNAKARAN R, FLOCK G AND GIGUERE V (1994). Identification of RVR, a 
novel orphan nuclear receptor that acts as a negative transcriptional regulator. Mol Endocrino/8: 1234-1244.

RUBIN J AND ROBERTSON A (1975). The tip of the Dictyostelium discoideum pseudoplasmodium as an organizer. J Embryol Exp Morpho/33: 227-241.

SCHNITZLER G R, FISCHER W H AND FIRTEL R A (1994). Cloning and characterization of the $\mathrm{G}$-box binding factor, an essential component of the developmental switch between early and late development in Dictyostelium. Genes Deve/8: 502-514.

SMITHE AND WILLIAMS K (1980). Evidence for tip control of the «slug/fruit» switch in slugs of Dictyostelium discoideum. J Embryol Exp Morpho/57: 233-240.
VERKERKE-VAN WIJK I, FUKUZAWA M, DEVREOTES P N AND SCHAAP P (2001). Adenylyl cyclase A expression is tip-specific in Dictyostelium slugs and directs StatA nuclear translocation and CudA gene expression. Dev Bio/234: 151-160.

WANG H Y AND WILLIAMS J G (2009). Identification of a target for CudA, the transcription factor which directs formation of the Dictyostelium tip organiser. Int J Dev Bio/54: 161-165.

YAMADA Y, WANG H Y, FUKUZAWA M, BARTON, G J AND WILLIAMS, J G (2008). A new family of transcription factors Development 135: 3093-3101.

\section{Further Related Reading, published previously in the Int. J. Dev. Biol.}

See our recent Special Issue Placenta edited by Joan S. Hunt and Kent L. Thornburg at: http://www.ijdb.ehu.es/web/contents.php?vol=54\&issue=2-3

Identification of a target for CudA, the transcription factor which directs formation of the Dictyostelium tip organiser Hong-Yu Wang and Jeffrey G. Williams

Int. J. Dev. Biol. (2010) 54: 161-165

Expression of zinc transporter family genes in Dictyostelium

Nobuya Sunaga, Meri Monna, Nao Shimada, Mai Tsukamoto and Takefumi Kawata

Int. J. Dev. Biol. (2008) 52: 377-381

Analysis of a homologue of the adducin head gene which is a potential target for the Dictyostelium STAT protein Dd-STATa Ryota Aoshima, Rieko Hiraoka, Nao Shimada and Takefumi Kawata

Int. J. Dev. Biol. (2006) 50: 523-532

Identification of new modes of Dd-STATa regulation of gene expression in Dictyostelium by in situ hybridisation Nao Shimida, Mineko Maeda, Hideko Urushihara and Takefumi Kawata

Int. J. Dev. Biol. (2004) 48: 679-682

Expression and role of adenylyl cyclases during late development in Dictyostelium discoideum

E Alvarez-Curto, ME Meima, P Schaap

Int. J. Dev. Biol. (2001) 45: S147-S148

The avian organizer

T Boettger, $\mathrm{H}$ Knoetgen, L Wittler and $M$ Kessel

Int. J. Dev. Biol. (2001) 45: 281-287

Evolution of the organizer and the chordate body plan

J Gerhart

Int. J. Dev. Biol. (2001) 45: 133-153

The MADS-box transcription factor SRFA regulates different aspects of Dictyostelium discoideum development

N Moreno, JJ Vicente, R Escalante, L Sastre

Int. J. Dev. Biol. (2001) 45: S117-S118

Cell cycle phase, cellular Ca2+ and development in Dictyostelium discoideum M Azhar, P K Kennady, G Pande, M Espiritu, W Holloman, D Brazill, R H Gomer and V Nanjundiah

Int. J. Dev. Biol. (2001) 45: 405-414

A cell type-specific effect of calcium on pattern formation and differentiation in dictyostelium discoideum

R Baskar, P Chhabra, P Mascarenhas and V Nanjundiah

Int. J. Dev. Biol. (2000) 44: 491-498

Regulation of cell differentiation and pattern formation in Dictyostelium development I Takeuchi, M Tasaka, K Okamoto and Y Maeda Int. J. Dev. Biol. (1994) 38: 311-319

5 yr ISI Impact Factor (2009) $=3.253$

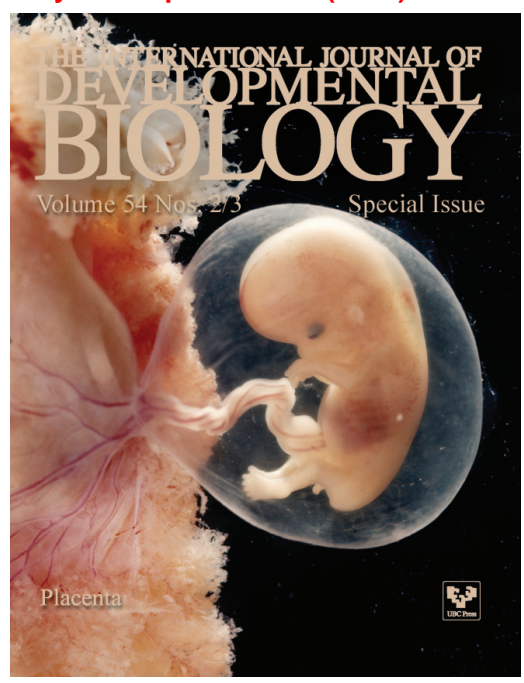

